\title{
THE CONFORMAL ROTATION NUMBER
}

\author{
Osamu Kobayashi
}

\begin{abstract}
The rotation number of a planar closed curve is the total curvature divided by $2 \pi$. This is a regular homotopy invariant of the curve. We shall generalize the rotation number to a curve on a closed surface using conformal geometry of ambient surface. This conformal rotational number is not integral in general. We shall show the fractional part is relevant to harmonic 1 -forms of the surface.
\end{abstract}

\section{Introduction}

Let $M$ be a connected oriented closed surface with a Riemannian metric $g$. The conformal Laplacian $L_{g}$ is defined as $L_{g} u=-\Delta_{g} u+K_{g}$, where $K_{g}$ is the Gauss curvature of $g$. If we denote by $G_{p}$ a Green function of $L_{g}$ with pole at $p \in M$, we have a flat surface $\left(M_{p}, g_{p}\right)=\left(M \backslash\{p\}, e^{2 G_{p}} g\right)$. Then for a regular closed curve $\gamma: S^{1} \rightarrow M_{p}$ we set $r(\gamma, p)=\frac{1}{2 \pi} \int_{\gamma} \kappa d s$, which will be called relative rotation number or conformal rotation number, where $\kappa$ is the curvature of $\gamma$ with respect to $g_{p}$. This is a conformally invariant with repect to $g$, and a regular homotopy invariant of $\gamma$, but not in general integer valued. We think of $r(\gamma, p)$ as a function in $p \in M$. It turns out that $r(\gamma, p)$ is not continuous for $p \in \gamma\left(S^{1}\right)$ if $\chi(M) \neq 0$, but the differential extends smoothly on $M$, and we write $\alpha_{\gamma}$ for this 1 -form. The main result of this paper is the following.

THEOREM 1.1. If $\chi(M)<0$, then $\frac{1}{\chi(M)} \alpha_{\gamma}$ is the harmonic form whose de Rham cohomology class is the Poincaré dual of $\gamma$.

We shall also explain the relation between our conformal rotation number and Reinhart's $\bmod \chi(M)$ invariant $([3])$.

2010 Mathematics Subject Classification. 53A30.

Key words and phrases. conformal geometry, regular homotopy.

Received March 25, 2014; revised July 31, 2014. 


\section{Conformal Laplacian of a surface}

Let $(M, g)$ be a connected closed surface and $K_{g}$ the Gauss curvature of $g$. The conformal Laplacian is defined as $L_{g} u=-\Delta_{g} u+K_{g}$.

LEMMA 2.1. If $\tilde{g}=e^{2 u} g$, then we have

(1) $L_{g} u=e^{2 u} K_{\tilde{g}}$;

(2) $L_{g} v=e^{2 u} L_{\tilde{g}}(v-u)$;

(3) $\Delta_{g} v=e^{2 u} \Delta_{\tilde{g}} v$;

(4) $\int_{M} L_{g} u d \mu_{g}=2 \chi(M)$;

(5) $u-v=$ const if $L_{g} u=L_{g} v$.

Proof. (4) is the Gauss-Bonnet theorem. The others are easily verified.

Now we assume moreover that $M$ is oriented. $\kappa_{g}$ denotes the curvature of a regular curve $\gamma$.

Lemma 2.2. Suppose $\tilde{g}=e^{2 u} g$ and $\gamma$ is a regular curve.

(1) $\int_{\gamma} \kappa_{\tilde{g}} d s_{\tilde{g}}-\int_{\gamma} \kappa_{g} d s_{g}=-\int_{\gamma}\left(\partial_{\nu} u\right) d s_{g}$, where $v$ is the unit normal vector of $\gamma$.

(2) If $\gamma=\partial U$ then $\int_{\gamma} \kappa_{\tilde{g}} d s_{\tilde{g}}-\int_{\gamma} \kappa_{g} d s_{g}=\int_{U}\left(\Delta_{g} u\right) d \mu_{g}$.

(3) If $\gamma=\partial U$ then $\int_{\gamma} \kappa_{\tilde{g}} d s_{\tilde{g}}=2 \pi \chi(\bar{U})-\int_{U}\left(L_{g} u\right) d \mu_{g}$.

Proof. (1) is a direct calculation. (2) follows from (1). (3) is GaussBonnet.

Definition 2.3. $G_{p} \in C^{\infty}\left(M_{p}\right)$ is called a Green function of $L_{g}$ with pole at $p \in M$ if $L_{g} G_{p}=a \delta_{p}^{g}$ for some constant $a$, where $\delta_{p}^{g}$ is the Dirac $\delta$-function at $p$ with repect to the metric $g$.

LEMMA 2.4 .

(1) $a=2 \pi \chi(M)$.

(2) $G_{p}$ is unique up to an additive constant.

(3) $G_{p}-u$ is a Green function of $e^{2 u} g$.

Proof. From Lemma 2.1.

We remark that $G_{p}$ has no pole at $p$ if $\chi(M)=0$.

COROLlaRY 2.5. $\left(M_{p}, g_{p}\right)$ is flat and its homothety class depends only on the conformal class of $(M, g)$.

Proposition 2.6. There is a Green function $G_{p}$, and $G_{p}(x)+\chi(M) \log d(x, p)$ is continuous at $p$. 
Proof. We have a metric $\tilde{g}=e^{2 \lambda} g$ which is flat near $p$. Let $u \in C^{\infty}\left(M_{p}\right)$ be a function such that $u(x)=-\chi(M) \log \tilde{d}(x, p)$ near $p$ and we have $-\Delta_{\tilde{g}} u=$ $2 \pi \chi(M) \delta_{p}^{\tilde{g}}$ near $p$. Put

$$
v(x)= \begin{cases}0 & x=p \\ \Delta_{\tilde{g}} u & \text { otherwise. }\end{cases}
$$

Then $v \in C^{\infty}(M)$ and $\Delta_{\tilde{g}} u=v-2 \pi \chi(M) \delta_{p}^{\tilde{g}}$. It follows from the Gauss-Bonnet theorem that $\int_{M}\left(K_{\tilde{g}}-v\right) d \mu_{\tilde{g}}=0$. Hence we have $w \in C^{\infty}(M)$ such that $\Delta_{\tilde{g}} w=$ $K_{\tilde{g}}-v$, and $G_{p}=u+w-\lambda \in C^{\infty}(M \backslash\{p\})$ is the desired Green function.

We will give a proof of the following classical theorem.

Proposition 2.7. Any metric $g$ is conformal to a metric of constant curvature.

Proof. Case $\chi(M)=0$ : The Poisson equation $-\Delta_{g} u+K_{g}=0$ is solvable. Case $\chi(M)<0$ : Let $u \in C^{\infty}(M)$ be a solution of $L_{g} u=2 \pi \chi(M) / \int_{M} d \mu_{g}$, and put $u_{+}=u-\min _{x} u(x)$ and $u_{-}=u-\max _{x} u(x)$. Because $\chi(M)<0$ the method of sub- and super-solutions (pp. 35-36 of [2]) is applicable, and we get $v \in C^{\infty}(M)$ such that $L_{g} v=e^{2 v} \cdot 2 \pi \chi(M) / \int_{M} d \mu_{g}$. Case $\chi(M)>0$ : Take $p \in M$ and consider $\left(M_{p}, g_{p}\right)$. We have $g_{p}=\lambda d(p, x)^{-2 \chi(M)} g$, where $\lambda$ is a fucntion continuous at $p$. Hence $\left(M_{p}, g_{p}\right)$ is a complete flat surface with one end because $\chi(M)>0$. Therefore $\left(M_{p}, g_{p}\right)$ is isometric to either $\left(\mathbf{R}^{2}, g_{0}\right)$ or $\left(S^{1} \times \mathbf{R} / \pm 1, g_{0}\right)$. That is, $(M, g)$ is conformal to $\left(S^{2}, g_{0}\right)$ or $\left(\mathbf{R P}^{2}, g_{0}\right)$.

We set $G(x, y)=G_{x}(y), x \neq y \in M$, and call it a Green kernel of $L_{g}$.

Proposition 2.8. We can choose a Green kernel so that $G(x, y)=G(y, x)$.

Proof. Suppose $\tilde{g}=e^{2 u} g$ has constant Gauss curvature $\tilde{K}$. Take a Green function $\tilde{G}_{p}$ of $L_{\tilde{g}}$. Note that $\tilde{G}_{p}$ is integrable and set $G^{\prime}(x, y)=\tilde{G}_{x}(y)-$ $\int_{M} G_{x}(y) d y / \int_{M} d y$. It is not hard to see that $G^{\prime}(x, y)=G^{\prime}(y, x) . G(x, y)=$ $G^{\prime}(x, y)+u(x)+u(y)$ is the desired Green kernel.

Remark 2.9. Let $0=\lambda_{0}<\lambda_{1} \leq \lambda_{2} \leq \cdots$ be the eigenvalues of $-\Delta_{\tilde{g}}$, and $\phi_{i}$ be eigenfunction with eigenvalue $\lambda_{i}$ such that $\int_{M} \phi_{i} \phi_{j}=\delta_{i j}$. Then $G^{\prime}(x, y)=$ $2 \pi \chi(M) \sum_{k>0} \frac{1}{\lambda_{k}} \phi_{k}(x) \phi_{k}(y)$. (cf. [1].)

In the case of nonpositive Euler characteristic $\left(M_{p}, g_{p}\right)$ is no longer complete, that is, $p$ may be regarded as a singular point rather than a point at infinity. The following describes a local picture around $p$.

Proposition 2.10. Suppose $\chi(M) \leq 0$. Then there are neighborhoods $U$ of $p$ and $V$ of 0 in $\mathbf{R}^{2}$, and a mapping $f: U \rightarrow V$ such that $f$ is a ramified covering of degree $1-\chi(M)$ branched at $p$ with $f(p)=0$ and $g_{p}=f^{*} g_{0}$, where $g_{0}$ is the 
Euclidean metric. In particular, there is a local coordinates $x^{1}$ and $x^{2}$ around $p$ such that $g_{p}=|x|^{-2 \chi(M)} \delta_{i j} d x^{i} d x^{j}$ near $p$.

Proof. Routine and omitted.

\section{Rotation number relative to a reference point}

Let $(M, g)$ be as before and $\gamma: S^{1} \rightarrow M$ be a regular closed curve. For $p \in M \backslash \gamma\left(S^{1}\right)$ we set

$$
r(\gamma, p)=\frac{1}{2 \pi} \int_{\gamma} \kappa d s
$$

where the curvature $\kappa$ of $\gamma$ and the line element $d s$ are with respect to the flat metric $g_{p}$.

LEMMA 3.1.

(1) $r(\gamma, p)$ depends only on the conformal class of $g$.

(2) If $\tilde{\gamma}$ is regularly homotopic to $\gamma$ in $M_{p}$, then $r(\tilde{\gamma}, p)=r(\gamma, p)$.

(3) Suppose that $\tilde{\gamma}$ is regularly homotopic to $\gamma$ in $M$, and that in the course of homotopy the point $p$ is passed once in such a way that $p$ is in the left of $\gamma$ and in the right of $\tilde{\gamma}$. Then $r(\tilde{\gamma}, p)=r(\gamma, p)+\chi(M)$.

Proof. (1) Since $\kappa d s$ is invariant under homothety of ambient metric, the result follows from Corollary 2.5. (2) We have only to consider a regular homotopy whose support is very small. Then the result is evident because $g_{p}$ is flat. (3) Let $D$ be a sufficiently small disk around $p$ with smooth boundary $c=\partial D$. Then it is easy to see that $r(\tilde{\gamma}, p)-r(\gamma, p)=1-r(c, p)$. On the other hand we have, from Lemma $2.2(3), r(c, p)=1-\chi(M)$.

Corollary 3.2. $\quad r_{\gamma}: M \rightarrow \mathbf{R} / \chi(M) \mathbf{Z} ; \quad r_{\gamma}(p)=r(\gamma, p) \bmod \chi(M)$ is welldefined and smooth.

Proof. From Lemma $2.2(1)$ and Proposition 2.8 it follows that $r(\gamma, p)$ is smooth in $p \notin \gamma\left(S^{1}\right)$. The result then follows from Lemma 3.1.

Proposition 3.3. If $\gamma$ is null homologous in $H_{1}(M, \mathbf{Z})$, then

(1) $r(\gamma, p) \in \mathbf{Z}$

(2) $r(\gamma, p)$, as a function of $p$, is locally constant for $p \notin \gamma\left(S^{1}\right)$;

(3) $r(\gamma):=r(\gamma, p) \bmod \chi(M)$ is well-defined.

Proof. Let $q$ be a point on $\gamma$. Since $g_{p}$ is flat, we have holonomy $\varphi$ : $\pi_{1}\left(M_{p}, q\right) \rightarrow S O(2)=U(1)$. This is explicitly given as $\varphi([c])=\exp (-2 \pi r(c, p))$. Since $U(1)$ is Abelian, and $H_{1}\left(M_{p}, \mathbf{Z}\right)=H_{1}(M, \mathbf{Z}), \varphi$ induces a homomorphism $\varphi: H_{1}(M, \mathbf{Z}) \rightarrow U(1)$. Hence $\varphi([\gamma])=1$, which implies (1). From Corollary 3.2 we get (2) because of (1). Then (3) follows from Lemma 3.1 (3). 
For a regular closed curve $\gamma$ on $M=S^{2}$ we have $r(\gamma)=0$ or $1 \bmod 2$. It is easy to see that this is a complete invariant of regular homotopy on $S^{2}$ (see also [4]).

We note that the above definitions and arguments make sense for multiple curve $\gamma: S^{1} \cup \cdots \cup S^{1} \rightarrow M$. Thus we have

Corollary 3.4. If $\gamma$ is homologous to $\tilde{\gamma}$ in $H_{1}(M, \mathbf{Z}), r(\gamma, p)-r(\tilde{\gamma}, p) \in \mathbf{Z}$ for $p \in M \backslash(\gamma \cup \tilde{\gamma})$, and its residue class modulo $\chi(M)$, which will be denoted by $r(\gamma, \tilde{\gamma})$, is independent of $p$.

Let $\mu_{1}, \ldots, \mu_{2 g}$ be regular curves which generate $\pi_{1}(M)$, where $g=$ $1-\chi(M) / 2$. Then they constitute also a basis for $H_{1}(M, \mathbf{Z})$. Hence for $\gamma$, we have $n_{i} \in \mathbf{Z}$ such that $\gamma$ is homologous to $\tilde{\gamma}=\sum n_{i} \mu_{i}$. The rotation number defined by Reinhart [3] is $r(\gamma, \tilde{\gamma})$ in our terminology.

Suppose $N$ is a compact surface with boundary and $f: N \rightarrow M$ is an immersion. Obviously $c=f \mid \partial N$ is null homologous. In this setting we have a simple formula.

LEMMA 3.5. $r(c, p)+m_{p} \chi(M)=\chi(N)$, where $m_{p}=\# f^{-1}(p)$.

Proof is easy and omitted.

Corollary 3.6. If $\chi(M) \geq 0$ then $\chi(N) \geq r(c, p)$. If $\chi(M) \leq 0$ then $\chi(N) \leq r(c, p)$.

\section{Proof of Theorem 1.1}

From Corollary 3.2 we have $r(\gamma, \cdot) \in C^{\infty}(M \backslash \gamma)$. Thus $\alpha_{\gamma}=d r(\gamma, \cdot)=d r_{\gamma}$ extends smoothly on $M$ as a closed 1-form. Moreover Lemma 3.1 (3) yields the following.

$$
\int_{c} \alpha_{\gamma}=\chi(M) \gamma \cdot c
$$

where $c$ is a smooth 1-cycle and "." in the right hand side is the homology intersection. Therefore if $\chi(M)<0, \frac{1}{\chi(M)}\left[\alpha_{\gamma}\right] \in H_{D R}^{1}(M)$ is the Poincare dual of
the cycle $\gamma$.

The key of the proof is Proposition 2.8. We write $K$ for $K_{g}$.

$$
-\Delta_{x} G_{p}(x)+K(x)=0 \text { if } p \neq x .
$$

We see from Proposition 2.8 that

$$
-\Delta_{p} G_{p}(x)+K(p)=0 \text { if } p \neq x
$$


Therefore $v$ being the unit normal vector of $\gamma$, we have

$$
-\Delta_{p} \partial_{v} G_{p}(x)=-\partial_{v} \Delta_{p} G_{p}(x)=\partial_{v}\left(-\Delta_{p} G_{p}(x)+K(p)\right)=0 \text { if } p \neq x .
$$

This together with Lemma 2.2 (1) shows that $r(\gamma, \cdot)$ is harmonic in $M \backslash \gamma$, and hence $\alpha_{y}$ is harmonic.

\section{Supplementary remarks}

Regular homotopy of closed curves is completely described by Smale [4] in terms of algebraic topology. We are interested in differential geometric interpretation of regular homotopy. Our conformal rotation number is not a complete invariant of regular homotopy. There is another non-trivial regular homotopy invariant $t(\gamma)$ (see [5]). It is of interest to understand $t(\gamma)$ from differential geometric point of view.

We distinguish the term "rotation number" from "winding number." The winding number is also generalized to a curve $\gamma$ on a surface $M$, which is given as

$$
w\left(\gamma, p_{0}, p_{\infty}\right)=-\frac{1}{\chi(M)}\left(r\left(\gamma, p_{0}\right)-r\left(\gamma, p_{\infty}\right)\right), \quad p_{0}, p_{\infty} \in M \backslash \gamma,
$$

if $\chi(M) \neq 0$.

\section{REFERENCES}

[1] I. Chavel, Eigenvalues in Riemannian geometry, Academic Press, 1984.

[2] J. L. KaZdan and F. W. Warner, Curvature functions for compact 2-manifolds, Ann. of Math. 99 (1974), 14-47.

[3] B. L. ReInhaRT, The winding number on two manifolds, Ann. Inst. Fourier, Grenoble 10 (1969), 271-283.

[4] S. Smale, Regular curves on riemannian manifolds, Trans. Amer. Math. Soc. 87 (1958), 492-512.

[5] H. Tanio and O. Kobayashi, Rotation numbers for curves on torus, Geom. Dedicata 61 (1996), 1-9.

Osamu Kobayashi

Department of Mathematics

OSAKA UNIVERSITY, TOYONAKA

OSAKA 560-0043

JAPAN

E-mail: kobayashi@math.sci.osaka-u.ac.jp 